\title{
Reconciling the Role of Serotonin in Behavioral Inhibition and Aversion: Acute Tryptophan Depletion Abolishes Punishment-Induced Inhibition in Humans
}

\author{
Molly J. Crockett, Luke Clark, and Trevor W. Robbins \\ Behavioral and Clinical Neuroscience Institute, Department of Experimental Psychology, University of Cambridge, Cambridge CB2 3EB, United Kingdom
}

\begin{abstract}
The neuromodulator serotonin has been implicated in a large number of affective and executive functions, but its precise contribution to motivation remains unclear. One influential hypothesis has implicated serotonin in aversive processing; another has proposed a more general role for serotonin in behavioral inhibition. Because behavioral inhibition is a prepotent reaction to aversive outcomes, it has been a challenge to reconcile these two accounts. Here, we show that serotonin is critical for punishment-induced inhibition but not overall motor response inhibition or reporting aversive outcomes. We used acute tryptophan depletion to temporarily lower brain serotonin in healthy human volunteers as they completed a novel task designed to obtain separate measures of motor response inhibition, punishment-induced inhibition, and sensitivity to aversive outcomes. After a placebo treatment, participants were slower to respond under punishment conditions compared with reward conditions. Tryptophan depletion abolished this punishment-induced inhibition without affecting overall motor response inhibition or the ability to adjust response bias in line with punishment contingencies. The magnitude of reduction in punishment-induced inhibition depended on the degree to which tryptophan depletion reduced plasma tryptophan levels. These findings extend and clarify previous research on the role of serotonin in aversive processing and behavioral inhibition and fit with current theorizing on the involvement of serotonin in predicting aversive outcomes.
\end{abstract}

\section{Introduction}

Few would disagree that serotonin is involved in aversive processing, but the nature of this function remains unclear. Aversive events (such as inescapable shocks) activate serotonin-releasing neurons (Takase et al., 2004), and animals with depleted serotonin levels show reduced behavioral suppression to cues and contexts predictive of punishment (for review, see Soubrié, 1986). Such results suggest that the general function of serotonin in motivation is to encode aversive outcomes. However, other evidence points in the opposite direction: boosting serotonin with selective serotonin reuptake inhibitors (SSRIs) is a major therapy for depression, and temporarily lowering serotonin in humans appears to enhance aversive processing in a variety of cognitive tasks (Cools et al., 2008b).

Another influential account of serotonin function pointed out that the observed reductions in behavioral suppression after serotonin depletion could be explained by an impairment in behavioral inhibition rather than a specific reduction in sensitivity to

Received May 29, 2009; revised Aug. 6, 2009; accepted Aug. 7, 2009.

This work was completed within the University of Cambridge Behavioural and Clinical Neuroscience Institute, funded by a joint award from the Medical Research Council and the Wellcome Trust. Additional funding for this study came from Wellcome Trust Programme Grant 076274/Z/04/Z awarded to T.W.R., B. J. Everitt, A. C. Roberts, and B. J. Sahakian. M.J.C. is supported by the Gates Cambridge Trust. We thank the nurses and administrative staff at the Wellcome Trust Clinical Research Facility (Addenbrooke's Hospital, Cambridge, UK), Oliver J. Robinson, Roshan Cools, Yevheniia Mikheenko, Angie Kehagia, and all participants.

Correspondence should be addressed to Molly J. Crockett, Department of Experimental Psychology, University of Cambridge, Downing Street, Cambridge CB2 3EB, UK. E-mail: mc536@cam.ac.uk.

D01:10.1523/JNEUROSCI.2513-09.2009

Copyright $\odot 2009$ Society for Neuroscience $\quad 0270-6474 / 09 / 2911993-07 \$ 15.00 / 0$ aversive outcomes (Soubrié, 1986). Thus, the main role of serotonin in motivation may be to generally suppress or inhibit behavior. However, because the prepotent reaction to aversive outcomes is behavioral inhibition (LeDoux, 1996; Gray and McNaughton, 2000), it is difficult to disentangle the aversion account of serotonin function from the inhibition account. Recent work in humans and rats has examined the effects of serotoninergic manipulations on motor response inhibition under conditions of non-punishment. However, these studies have failed to detect effects of serotonin manipulations on motor response inhibition (Clark et al., 2005; Eagle et al., 2009).

This apparent conflict between the behavioral inhibition hypothesis of serotonin (Soubrié, 1986) and the lack of experimental evidence implicating serotonin in general motor response inhibition may be reconciled by considering that the link between serotonin and inhibition is specific to aversive contexts. Although most of the research investigating the role of serotonin in behavioral inhibition in animals has measured inhibition in response to punishment and nonreward, studies in humans have primarily focused on non-reinforced measures of motor response inhibition. Here, we sought to bridge the gap between these two literatures by simultaneously investigating the role of serotonin in two forms of inhibition: punishment-induced inhibition, which we defined as the general suppression of responding in aversive contexts (Gray and McNaughton, 2000), and motor response inhibition, which we defined as the intentional inhibition of inappropriate motor responses (Aron et al., 2004).

In the current experiment, we used acute tryptophan depletion (Young et al., 1985) to temporarily lower serotonin levels in 
healthy human volunteers and tested the effects of tryptophan depletion on behavior in a novel task designed to obtain separate measures of motor response inhibition, punishment-induced inhibition, and sensitivity to aversive outcomes.

\section{Materials and Methods}

Participants. The experimental protocol was approved by the Norfolk Research Ethics Committee. Twenty-two healthy subjects (eight males; mean age, 25.6 years) were screened for neurological and psychiatric disorders and gave written informed consent before participating in the study. Exclusion criteria included history of cardiac, hepatic, renal, pulmonary, neurological, psychiatric, or gastrointestinal disorders, medication/drug use, and personal or family history of major depression or bipolar affective disorder. Participants were financially compensated for participating. After the screening interview, participants were assigned to receive either the tryptophan depleting drink or the placebo mixture on the first session in a double-blind, counterbalanced order. The reinforced go/no-go task was administered as part of a larger neuropsychological testing battery (data to be published separately by M.J.C. and T.W.R.).

General procedure. We used acute tryptophan depletion to temporarily lower serotonin levels in a double-blind, placebo-controlled, withinsubjects, counterbalanced design. Tryptophan is the amino acid precursor of serotonin, and dietary depletion of tryptophan causes a rapid decrease in the synthesis and release of brain serotonin, as confirmed by brain tissue analysis in rats (Biggio et al., 1974; Gessa et al., 1974; Moja et al., 1989; Ardis et al., 2009), microdialysis in rats (Stancampiano et al., 1997; Fadda et al., 2000a,b; van der Stelt et al., 2004) (but see van der Plasse et al., 2007), and positron emission tomography (PET) in humans (Nishizawa et al., 1997). In the tryptophan depletion procedure, tryptophan was depleted by ingestion of a liquid amino acid load that did not contain tryptophan but did include other large neutral amino acids (for content of amino acid mixtures, see supplemental methods, available at www.jneurosci.org).

Participants completed a battery of neuropsychological tests on two separate sessions, separated by at least 1 week. Participants were asked to abstain from food, alcohol, and caffeine from midnight before each session. Testing sessions commenced in the morning (between 8:30 A.M. and 10:30 A.M.). On arrival, participants completed a baseline self-report mood questionnaire, gave a blood sample, and ingested either the placebo or the tryptophan depleting amino acid drink. After a resting period of $\sim 5.5 \mathrm{~h}$ to achieve stable and low tryptophan levels (Carpenter et al., 1998), participants completed a second self-report mood questionnaire, gave a second blood sample, and completed the test battery. Self-report mood was assessed at three other time points during the battery.

Behavioral task. To obtain independent measures of executive inhibition, punishment-induced inhibition, and sensitivity to aversive outcomes, we adapted the classic go/no-go procedure in several ways. First, we created a stimulus set that allowed us to manipulate task difficulty. The stimuli were checkerboards composed of blue and yellow squares. Throughout the task, participants were instructed to press a button ("go") if the "target color" was in the majority and to not respond (nogo) if the target color was in the minority. The target color was blue for half the participants, and yellow for the other half. Varying the ratio of blue to yellow squares allowed us to create easy and difficult stimuli; easy stimuli had a high ratio of target/nontarget color (e.g., 16 blue, 9 yellow), whereas difficult stimuli had a small ratio of target/nontarget color (e.g., 13 blue, 12 yellow). All task conditions contained $50 \%$ go trials and $50 \%$ no-go trials, split evenly between easy and difficult stimuli (for sample stimuli, see supplemental Fig. S1, available at www.jneurosci.org as supplemental material).

\section{b Reward-NoGo}

\begin{tabular}{c|l|c|c|}
\multicolumn{2}{c|}{} & \multicolumn{2}{c}{ Stimulus } \\
\cline { 2 - 4 } Response & Go & No-go \\
\cline { 2 - 4 } & Go & $\begin{array}{c}+1 \\
\text { point }\end{array}$ & 0 points \\
\cline { 2 - 4 } & No-go & 0 points & $\begin{array}{c}+10 \\
\text { points }\end{array}$ \\
\hline
\end{tabular}

\section{d Punish-NoGo}

\begin{tabular}{c|l|c|c|}
\multicolumn{2}{c}{} & \multicolumn{3}{c}{ Stimulus } \\
\cline { 2 - 4 } Response & Go & No-go \\
\cline { 2 - 4 } & Go points & -1 point \\
\cline { 2 - 4 } No-go & $\begin{array}{c}-10 \\
\text { points }\end{array}$ & 0 points \\
\hline
\end{tabular}

In some of the task conditions, participants received feedback for their responses. Sometimes correct responses were rewarded: small rewards earned 1 point, a high tone, and a mildly happy face; large rewards earned 10 points, a flourishing tone, and an extremely happy face. Sometimes incorrect responses were punished: small punishments lost 1 point, a short buzzing tone, and a mildly angry face; large punishments lost 10 points, a long buzzing tone, and an extremely angry face. Throughout the task, feedback was presented for $1500 \mathrm{~ms}$. Points were exchangeable for bonus money at the end of the experiment. Faces were taken from the NimStim set of facial expressions (Tottenham et al., 2009).

To assess the influence of reward and punishment on performance, we crossed feedback type (reward, punishment) by response type (go, no-go) in four experimental conditions, each 36 trials in length. In the "reward-go" condition, correct go responses (hits) earned large rewards, whereas correct no-go responses (correct rejections) earned small rewards. In the complementary reward-no-go condition, correct no-go responses earned large rewards, whereas correct go responses earned small rewards. If reward signaling processes are intact, subjects should show a response bias toward go in the reward-go condition [i.e., faster reaction times (RTs), more go responses] and a response bias toward no-go in the reward-no-go condition (i.e., slower reaction times, fewer go responses). In the "punish-go" condition, incorrect go responses (commission errors) earned large punishments, whereas incorrect no-go responses (omission errors) earned small punishments. In the complementary "punish-no-go" condition, incorrect no-go responses earned large punishments, whereas incorrect go responses earned small punishments. If punishment signaling processes are intact, subjects should show a response bias away from go (toward no-go) in the punish-go condition and a response bias away from no-go (toward go) in the punishno-go condition. For a summary of response-outcome contingencies in the experimental conditions, see Figure 1.

The task began with 48 practice trials without feedback to minimize learning effects. Stimuli were presented for $2000 \mathrm{~ms}$, with an intertrial interval of $1500 \mathrm{~ms}$. The mean RT for correct responses was extracted from the practice session and set as the stimulus duration for the main task, to match task difficulty across participants and sessions.

The main task began with a neutral block of 36 trials to obtain a baseline RT. Next, participants completed the four experimental conditions (reward-go, reward-no-go, punish-go, punish-no-go) in counterbalanced order. The experimental conditions were separated by neutral blocks of 36 trials without feedback to allow response biases to return to baseline. At the start of each experimental condition, participants completed four guided practice trials to learn about the responseoutcome contingencies in the upcoming trials.

Analysis of behavioral data. In line with previous studies, we assessed motor response inhibition by examining the rate of commission errors 
(i.e., the proportion of trials on which the participant responded inappropriately to a no-go stimulus) across all experimental task conditions. In light of previous findings, we did not expect to see any effect of tryptophan depletion on commission error rates (Rubia et al., 2005; Evers et al., 2006b).

We assessed reward and punishment signaling by examining the adjustment of response bias (the tendency to favor go over no-go) according to response-outcome contingencies in the experimental conditions. Impaired reward signaling would be expected to reduce discrimination between large and small rewards, so subjects with impaired reward signaling should not prefer go over no-go in the reward-go condition and should not prefer no-go over go in the reward-no-go condition. Impaired punishment signaling would reduce discrimination between large and small punishments, so subjects with impaired punishment signaling should not prefer no-go over go in the punish-go condition and should not prefer go over no-go in the punish-no-go condition. Response bias was assessed by the natural $\log$ of $\beta$, or $\ln (\beta)$, from signal detection theory (Swets et al., 1961). Formulae for calculating $\ln (\beta)$ are widely available (Stanislaw and Toderov, 1999). Lower values of $\ln (\beta)$ indicate a stronger tendency toward go. Because performance on easy trials was nearly perfect, we restricted the analysis of commission errors and $\ln (\beta)$ to difficult trials only.

We assessed punishment-induced inhibition by examining RTs for correct go responses in punished conditions relative to rewarded and neutral conditions. This approach has been used in other studies of punishment-induced behavioral inhibition (Newman et al., 1997; Avila, 2001) and follows from the observation that the automatic response to aversive outcomes is to freeze or depress responding (LeDoux, 1996; Gray and McNaughton, 2000). Because this analysis was restricted to correct go trials, it is independent from the analysis of motor response inhibition, which was restricted to incorrect no-go trials. We reasoned that punishment-induced inhibition would result in slower responding in punished conditions, relative to rewarded and neutral conditions, as has been observed in previous studies (Newman et al., 1997; Avila, 2001). Preliminary analyses indicated that RTs for correct responses did not significantly differ across stimulus difficulty level, so we collapsed RTs across difficulty level by computing weighted averages. RTs were then converted to $z$-scores by normalizing against matched RTs in the first non-reinforced block.

The transformed raw data (commission error rates, response bias, normalized RTs) from the four experimental conditions were analyzed using repeated-measures ANOVAs (SPSS version 14), with treatment (tryptophan depletion, placebo), feedback (reward, punishment), and bias (go, no-go) as within-subjects factors. Treatment order and task condition order were initially included as between-subjects factors and dropped from subsequent analyses when nonsignificant.

In within-subject designs, the appropriate index of variation is not the SEMs but the standard error of the difference of the means (SED), which is used when one is interested in the relationship between variables rather than the variables themselves. The SED is therefore used in the figures as an index of variation. The SED is calculated using the formula provided by Cochran and Cox (1957, p 131):

$$
\mathrm{SED}=\{(2 \times \mathrm{MSe}) / n\}
$$

where MSe is mean square for the error, or residual, term, and $n$ is the number of observations made. The SED is the denominator for Student's $t$ test and also provides a visual method of comparing mean values in graphical depictions of within-subject designs.

Analysis of plasma samples. Blood samples $(10 \mathrm{ml})$ were analyzed to determine the total plasma tryptophan level and the ratio of tryptophan to other large neutral amino acids (TRP/ $\Sigma$ LNAA ratio). This ratio was calculated from the serum concentrations of total tryptophan divided by the sum of the large neutral amino acids (tyrosine, phenylalanine, valine, isoleucine, leucine) and is important because the uptake of tryptophan in the brain is strongly associated with the amounts of other competing large neutral amino acids attributable to nonspecific transport across the blood-brain barrier. Because individuals vary in their response to the tryptophan depleting treatment, the reduction in the TRP/ $\Sigma$ LNAA ratio

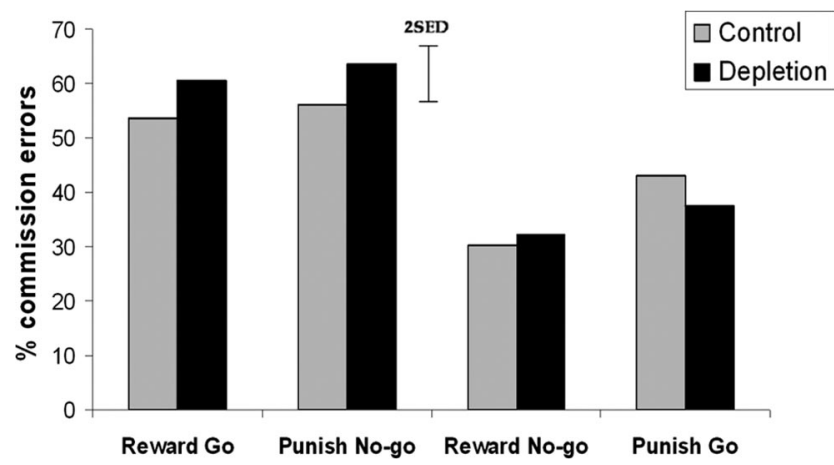

Figure 2. Effect of tryptophan depletion on motor response inhibition, measured by percentage of commission errors (incorrect go responses), in the four experimental conditions. Error bars depict the SED, the index of variation commonly used for within-subject designs.

after tryptophan depletion (compared with placebo) can be used as a proxy of degree of brain serotonin depletion; we used these values as a rough indicator of dose dependency of our behavioral effects (for details of plasma sample analysis, see supplemental methods, available at www.jneurosci.org).

\section{Results}

\section{Serotonin manipulation}

Tryptophan depletion resulted in significant reductions in both plasma tryptophan levels and the TRP/ $\Sigma$ LNAA ratio. A repeatedmeasures ANOVA revealed a significant two-way interaction between treatment (tryptophan depletion, placebo) and time point (baseline, $+5.5 \mathrm{~h}$ ), resulting from significant reductions in total tryptophan levels $\left(F_{(1,21)}=73.166, p<0.0001\right)$ and the TRP/ $\Sigma$ LNAA ratio $\left(F_{(1,21)}=45.968, p<0.0001\right), 5.5 \mathrm{~h}$ after tryptophan depletion relative to placebo. Simple effects analyses showed a significant decrease in plasma tryptophan levels $\left(t_{(21)}=\right.$ $15.648, p<0.0001)$ on the tryptophan depletion session, averaging $71 \%$. There was also a significant decrease in TRP/ $\Sigma$ LNAA ratios $\left(t_{(21)}=12.710, p<0.001\right)$ on the tryptophan depletion session, averaging $85 \%$. On the placebo session, plasma tryptophan levels increased by an average of $64 \%\left(t_{(21)}=-6.385, p<\right.$ $0.0001)$, and there was a trend toward increased TRP/ $\Sigma$ LNAA ratios $\left(t_{(21)}=-1.924, p=0.07\right)$, averaging $25 \%$.

Lowering serotonin does not affect motor response inhibition Across all task conditions, manipulating serotonin had no effect on motor response inhibition, as measured by commission error rates (main effect of treatment, $F_{(1,21)}=0.783, p=$ 0.386) (Fig. 2).

Motor response inhibition did vary as a function of responseoutcome contingencies. When the payoff schedule biased subjects' responding toward go (in reward-go and punish-no-go), participants made a higher proportion of commission errors than when the payoff schedule biased subjects' responding toward no-go (in reward-no-go and punish-go) (main effect of bias, $\left.F_{(1,21)}=14.676, p=0.001\right)$. However, this effect was not modulated by tryptophan depletion (treatment $\times$ bias interaction, $\left.F_{(1,21)}=2.779, p=0.11\right)$.

It is possible that our primary measure of motor response inhibition, commission errors, was not sufficiently sensitive to detect changes in response inhibition resulting from tryptophan depletion. We therefore analyzed RTs as a more sensitive measure of motor response inhibition. RTs were also sensitive to responseoutcome contingencies, in a similar manner to commission errors. When the payoff schedule biased responding toward go, RTs were 


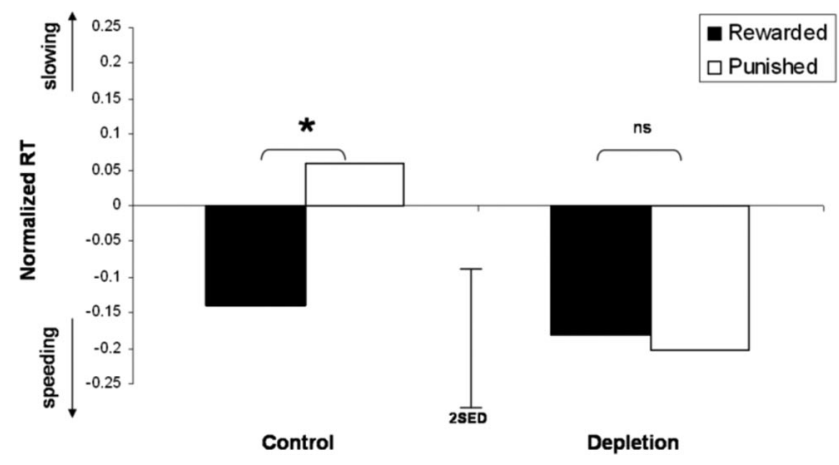

Figure 3. Effect of tryptophan depletion on punishment-induced inhibition, assessed by comparing RTs for correct go responses in punished conditions relative to rewarded conditions. All RTs were normalized against a neutral baseline. Error bars depict the SED. ${ }^{*} p<0.05$.

faster than when payoffs biased subjects' responding toward no-go (main effect of bias, $F_{(1,21)}=24.397, p<0.001$ ).

If lowering serotonin impairs motor response inhibition, then we might expect tryptophan depletion to produce a general speeding of RTs and/or to eliminate slowing in conditions biased away from go. Neither of these effects was observed. Tryptophan depletion did not induce general speeding in the experimental conditions (main effect of treatment on RT, $F_{(1,21)}=1.435, p=0.244$ ) or in the initial neutral block $\left(t_{(21)}=0.580, p=0.568\right)$. Furthermore, tryptophan depletion did not modulate the effect of bias on RTs (treatment $\times$ bias interaction, $\left.F_{(1,21)}=0.940, p=0.343\right)$.

\section{Lowering serotonin abolishes punishment-induced inhibition}

Our analysis of the RT data revealed a significant interaction between treatment and feedback on response speed $\left(F_{(1,21)}=\right.$ $5.210, p=0.033)$. On placebo, participants were slower to respond in punished conditions (mean $\pm \mathrm{SE}, 0.059 \pm 0.102$ ) than in rewarded conditions (mean $\pm \mathrm{SE},-0.139 \pm 0.127 ; t_{(21)}=-2.177$, $p=0.041$ ) (Fig. 3, left). However, this punishment-induced inhibition of responding was absent after tryptophan depletion; responses were not slower in punished conditions (mean $\pm \mathrm{SE}$, $-0.203 \pm 0.182$ ) compared with rewarded conditions (mean $\pm \mathrm{SE}$, $-0.181 \pm 0.149 ; t_{(21)}=0.254, p=0.802$ ) (Fig. 3, right).

The data indicate that tryptophan depletion likely abolished slowing in punished conditions rather than enhancing speeding in rewarded conditions; RTs in punished conditions showed a trend toward being significantly slower after placebo compared with tryptophan depletion $\left(t_{(21)}=-1.932, p=0.067\right)$, but there was no significant difference between RTs in rewarded conditions when comparing placebo with tryptophan depletion within subjects $\left(t_{(21)}=-0.305, p=0.764\right)$.

We next tested whether the reduction of slowing in punished conditions after tryptophan depletion was specific to trials that immediately followed the receipt of punishment. To do this, we broke down the RT data in the punished conditions into trials after correct responses (non-punished) and trials after incorrect responses (punished) and conducted a repeated-measures ANOVA with treatment and prior punishment as within-subjects factors. This analysis revealed a significant main effect of treatment $\left(F_{(1,21)}=4.806, p=\right.$ $0.040)$ but no significant interaction between treatment and prior punishment $\left(F_{(1,21)}=2.529, p=0.127\right)$. Across all trials in punished conditions, participants were faster to respond after tryptophan depletion compared with placebo. The general reduction in slowing across all trials in punished conditions after tryptophan depletion suggests that lowering serotonin dampened the behavioral effects of expectations of punishment, leading to faster responding.

Finally, we examined whether the reduction in punishmentinduced inhibition depended on the degree to which tryptophan depletion reduced serotonin levels. We performed a linear regression with the RT difference between punished and rewarded conditions as the dependent variable and change in plasma TRP/ ¿LNAA as the predictor variable. The effect of plasma TRP/ ¿LNAA on slowing in punished conditions, relative to rewarded conditions, was highly significant $\left(r=0.558, t_{(21)}=-2.992, p=\right.$ $0.007)$. Higher reductions in the ratio of plasma TRP/ $\Sigma$ LNAA (i.e., greater degree of depletion) corresponded to reduced slowing in punished conditions relative to rewarded conditions.

The plasma TRP/ $\Sigma$ LNAA data also indicate that tryptophan depletion abolished slowing in punished conditions rather than enhancing speeding in rewarded conditions. Greater biochemical depletion after tryptophan depletion significantly predicted larger reductions in slowing after tryptophan depletion (compared with placebo) in punished conditions $\left(t_{(21)}=2.853, p=0.011\right)$ but did not predict RT differences between the tryptophan depletion and placebo treatments in rewarded conditions $\left(t_{(21)}=\right.$ 1.087, $p=0.291$ ).

\section{Lowering serotonin does not affect sensitivity to aversive outcomes}

Given the potential role of serotonin in reporting aversive outcomes, we tested whether manipulating serotonin influenced sensitivity to punishments. First, we examined whether tryptophan depletion affected participants' ability to discriminate between aversive response-outcome contingencies. In the punish-go condition, the go response is punished more severely than the no-go response, so if punishment discrimination is intact, subjects should become biased toward the no-go response; in the punish-no-go condition, the no-go response is punished more severely than the go response, so if punishment discrimination is intact, subjects should become biased toward the go response.

If serotonin reports aversive outcomes, we might expect tryptophan depletion to reduce differences in response bias between the punish-go and punish-no-go conditions. This was not observed. We conducted a repeated-measures ANOVA on the response bias data from the punished conditions only. After both treatments, subjects were biased toward go in the punish-no-go condition and biased toward no-go in the punish-go condition (main effect of bias, $\left.F_{(1,21)}=11.853, p=0.002\right)$. There was no significant interaction between treatment and bias $\left(F_{(1,21)}=\right.$ $0.377, p=0.546)$. These results indicate that tryptophan depletion did not affect subjects' sensitivity to punishment contingencies; after tryptophan depletion, they were equally likely to favor the lesspunished response compared with placebo (Fig. 4).

We also examined whether tryptophan depletion altered the emotional impact of punishment on responding. Our analysis of RTs on trials after punishment versus trials after non-punishment showed a main effect of prior punishment $\left(F_{(1,21)}=23.024, p<\right.$ $0.001)$; subjects were slower to respond on trials that followed punishment (mean \pm SE, $0.303 \pm 0.177$ ) than on trials that followed non-punishment (mean $\pm \mathrm{SE},-0.234 \pm 0.131$ ), but the interaction between treatment and prior punishment was not significant $\left(F_{(1,21)}=2.529, p=0.127\right)$; tryptophan depletion did not abolish subjects' tendency to be slower on trials immediately after punishment compared with trials after non-punishment. Subjects' responses were significantly slower on trials after punishment compared with non-punishment on both tryptophan depletion $\left(t_{(21)}=-3.019, p=0.007\right)$ and placebo $\left(t_{(21)}=-4.085, p=0.001\right)$. 


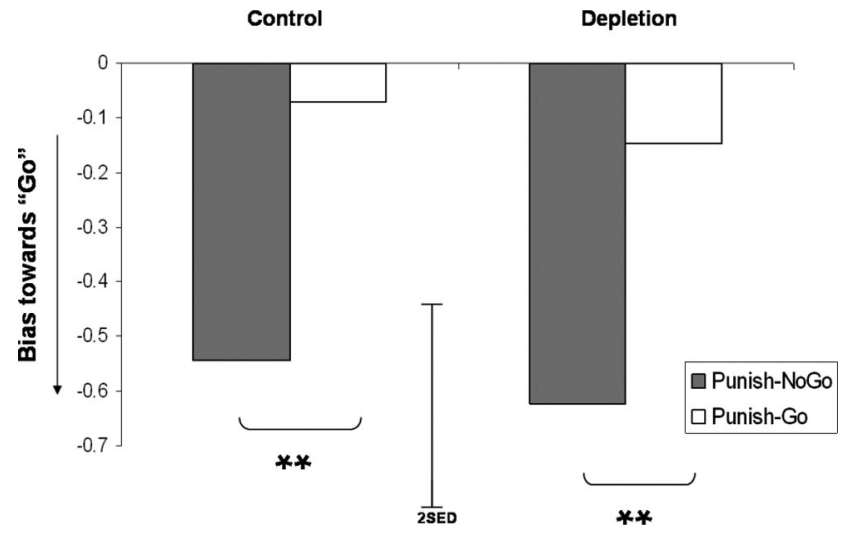

Figure 4. Effect of tryptophan depletion on punishment sensitivity, assessed by comparing response bias in the punish-go condition to the punish-no-go condition. Response bias was assessed by the natural $\log$ of $\beta$ from signal detection theory; more negative values indicate a bias toward go. If aversive signaling is intact, performance should be biased away from go in the punish-go condition and toward go in the punish-no-go condition. Error bars depict the SED. ${ }^{* *} p<0.01$.

Table 1. Summary of results

\begin{tabular}{lll}
\hline Function & Dependent measure & $\begin{array}{l}\text { Effect of tryptophan } \\
\text { depletion }\end{array}$ \\
\hline Motor response inhibition & $\begin{array}{c}\text { Commission errors across all task } \\
\text { conditions } \\
\text { Punishment-induced } \\
\text { inhibition }\end{array}$ & - \\
$\begin{array}{c}\text { Slowed RT in punished conditions } \\
\text { relative to rewarded conditions } \\
\text { magnitude }\end{array}$ & $\downarrow$ \\
$\begin{array}{c}\text { Reporting aversive outcome } \\
\text { occurrence }\end{array}$ & $\begin{array}{c}\text { Slowed RT on trials after punishment } \\
\text { punishment contingencies }\end{array}$ & - \\
& compared with trials after & - \\
\hline
\end{tabular}

\section{Discussion}

Temporarily lowering serotonin in humans led to a selective reduction in punishment-induced inhibition, without affecting motor response inhibition or sensitivity to aversive outcomes. The magnitude of reduction in punishment-induced inhibition depended on the degree to which tryptophan depletion reduced plasma tryptophan levels, suggesting dose dependency of this effect. Our results are summarized in Table 1.

Implications for the role of serotonin in behavioral inhibition These results provide perhaps the first demonstration in humans of a selective effect of manipulating serotonin on aversively motivated behavioral inhibition, suggesting that the concept of global behavioral inhibition as it stands is insufficiently precise to characterize the influence of serotonin on motivated behavior. By assessing behavioral suppression in response to aversive outcomes (punishment-induced inhibition) independently from general motor response inhibition, we were able to determine that lowering serotonin affected the former but not the latter form of inhibition. Breaking down the construct of behavioral inhibition in this way may help to clarify some of the apparent contradictions in the literature. For example, to account for the large body of research in experimental animals showing that interfering with the normal functioning of the serotonin system "releases" behavior suppressed by punishment (Geller et al., 1960; Stevens et al., 1969; Graeff and Schoenfeld, 1970; Wise et al., 1973; Tye et al., 1977, 1979; Thiébot et al., 1982; Cervo et al., 2000; Dekeyne et al., 2000; Graeff, 2002), it was proposed that serotonin plays a general role in inhibitory response control (Soubrié,
1986). However, studies in rats and humans have shown that manipulating serotonin does not always affect performance in tasks measuring general motor response inhibition such as the stop-signal reaction time task (Clark et al., 2005; Chamberlain et al., 2006; Bari et al., 2009; Eagle et al., 2009) and the go/no-go task (Rubia et al., 2005; Evers et al., 2006b). Thus, serotonin may play a role in behavioral inhibition specifically when aversive outcomes are expected (i.e., punishment-induced inhibition) rather than motor response inhibition in general.

An alternative account distinguishes the lack of effect of serotonin depletion on the cancellation of an already-initiated response from its impairment of action restraint, or waiting until a response is appropriate (Eagle et al., 2008, 2009). This formulation is consistent with the finding of intact stop-signal performance (Eagle et al., 2009) but impaired go/no-go acquisition and performance (Harrison et al., 1999) after global forebrain serotonin depletion in rats. However, the adverse effects of serotonin depletion on go/no-go performance in rats may depend on the aversive context of reward omission or timeout from positive reinforcement after commission errors (Gray and McNaughton, 2000). The lack of similar effects of serotonin depletion on human go/no-go performance (Rubia et al., 2005; Evers et al., 2006b) could be attributable to the absence of real rewards or punishments in these studies that might have induced an equivalent aversive state. The few studies that examined the effect of tryptophan depletion on reinforced go/no-go learning in humans produced mixed results and did not distinguish between motor response inhibition and punishment-induced inhibition (LeMarquand et al., 1998, 1999). Additional research in humans with tasks distinguishing action restraint, action cancellation, and punishment-induced inhibition is needed to reconcile these findings.

\section{Implications for the role of serotonin in aversive processing}

The reduction in punishment-induced inhibition after tryptophan depletion could potentially be explained by a role for serotonin in reporting aversive outcomes as they occur: if tryptophan depletion reduced sensitivity to punishing events, punishmentinduced inhibition would be impaired. However, our data imply that the involvement of serotonin in punishment-induced inhibition is not simply a reflection of reporting aversive outcomes. Tryptophan depletion did not affect subjects' ability to adjust response bias in line with punishment contingencies, suggesting that serotonin is not critical for processing the magnitude of punishments, nor did tryptophan depletion interfere with the tendency to be slower on trials after the receipt of punishment relative to trials after non-punishment. Instead, tryptophan depletion led to faster responding across all trials in punished conditions, not just trials that followed the receipt of punishment. This general reduction in behavioral inhibition during punished conditions after tryptophan depletion suggests that serotonin is involved in predicting aversive outcomes rather than reporting aversive outcomes as they occur because, in punished conditions, the expectation of punishment should remain constant across all trials.

If serotonin is involved in aversive prediction, one potential process model is that tryptophan depletion enhanced punishment prediction (Cools et al., 2008a), which potentiated quick responding to avoid punishment. However, this is unlikely given the pattern of results. If tryptophan depletion enhanced punishment prediction, we would have observed faster responding when the payoff structure biased responding toward go (punishno-go) but slower responding when the payoff structure biased responding toward no-go (punish-go). We found that tryptophan depletion abolished slowing in punished conditions, re- 
gardless of whether the payoff structure biased responding toward go or toward no-go (Fig. 3), which is more consistent with the interpretation that serotonin promotes behavioral inhibition in the face of aversive predictions, and lowering serotonin reduces this punishment-induced inhibition.

This interpretation is consistent with recent theorizing about the general role of serotonin in affective control (Dayan and Huys, 2009). According to this view, behavioral inhibition is a preprogrammed response to aversive predictions, putatively signaled by serotonin (Daw et al., 2002). Reducing serotonin function leads to a decline in aversive predictions, but predictions are difficult to measure; instead, researchers must infer predictions from measurable aspects of behavior and cognition. Herein lies one potential source of the many paradoxes that have been observed in the serotonin literature. For example, reducing serotonin function seems to reduce aversive processing in animals (Graeff, 2002) but enhance aversive processing in humans (Cools et al., 2008b). However, animal studies have used behavioral measures (e.g., lever pressing) to infer aversive predictions, whereas human studies have used cognitive measures (e.g., accuracy in choice procedures). If one general function of serotonin is to promote avoidance of potentially aversive stimuli (i.e., punishment-induced inhibition), then lowering serotonin would reduce avoidance of those stimuli. A reduction in behavioral avoidance of aversive stimuli is straightforward to measure and has been observed after global forebrain serotonin depletion in rats (Tye et al., 1977). Such a decline in behavioral avoidance of aversive stimuli could be interpreted as "reduced aversive processing," but what would be the consequences of reduced cognitive avoidance of aversive stimuli? One possibility is that lowering serotonin enhances cognitive engagement with aversive stimuli (Dayan and Huys, 2009). Enhanced cognitive engagement with aversive stimuli could result in more accurate detection, encoding, and prediction of aversive stimuli, all of which have been reported after manipulations that reduce serotonin function in humans (Klaassen et al., 2002; Murphy et al., 2002; Evers et al., 2006a; Browning et al., 2007; Cools et al., 2008a).

Potential neural mechanisms and methodological limitations There are a few limitations to the method we used to manipulate serotonin, acute tryptophan depletion. Although several studies in rats have shown that tryptophan depletion reduces serotonin levels in brain tissue (Biggio et al., 1974; Gessa et al., 1974; Moja et al., 1989), it is possible that low serotonin levels in the raphe may reduce $5-\mathrm{HT}_{1 \mathrm{~A}}$ receptor-mediated somatodendritic autoinhibition, thereby increasing the firing of raphe neurons and increasing the release of serotonin in projection regions. However, reduced levels of serotonin and its metabolite 5-HIAA have been reported in projection regions, including hippocampus and frontal cortex after tryptophan depletion (Ardis et al., 2009), and microdialysis studies have shown that tryptophan depletion reduces serotonin release in projection regions (Stancampiano et al., 1997; Fadda et al., 2000a,b; van der Stelt et al., 2004) (but see van der Plasse et al., 2007). It is more difficult to study directly whether tryptophan depletion alters serotonergic neurotransmission in humans, but there is evidence that the procedure reduces serotonin synthesis uniformly throughout the terminal fields of serotonergic neurons, as measured by PET (Nishizawa et al., 1997). Still, as a caveat, we allow that the effects of tryptophan depletion on serotonergic neurotransmission in humans are not entirely clear and warrant additional investigation.

Another limitation of tryptophan depletion is its subtlety in terms of degree of depletion; it is therefore not directly equivalent to profound global forebrain serotonin depletions, which has been used in animals. However, when it has been feasible to directly compare the different forms of serotonin depletion in animals and humans on a common procedure, such as stop-signal inhibition, qualitatively similar findings have been obtained (Clark et al., 2005; Eagle et al., 2009). The subtlety of the effects of tryptophan depletion may explain why our behavioral effects were limited to reaction times, which may be more sensitive to neuromodulation than response bias or commission errors (Rogers et al., 1999; Murphy et al., 2002; Cools et al., 2005).

Finally, it is worth asking what scientific value can be gleaned from such a global, neuroanatomically nonspecific manipulation. Although tryptophan depletion presumably affects multiple neural mechanisms, we investigated its effects on those mechanisms engaged by a behavioral task designed to test detailed hypotheses about the role of serotonin in aversion and inhibition. We find it remarkable that a global manipulation of serotonin had such a highly selective effect on motivated behavior, and our findings imply a more specific role for serotonin in motivated behavior than has been advocated by previous accounts. In addition, the effects of global manipulations are of biological and clinical interest, because genes and many drugs (e.g., SSRIs) exert widespread influences on brain function.

Because all of the punishments in our task were contingent on behavior, we are unable to determine from our data whether the reduction in punishment-induced inhibition by tryptophan depletion is mediated by pavlovian aversive reactions, instrumental avoidance reactions, or both. In other words, to what extent does serotonin modulate the prediction of pavlovian aversive outcomes versus instrumental aversive outcomes? Future studies might use punishments that are both contingent and noncontingent on behavior (Killcross et al., 1997) to dissociate the effects of serotonin manipulations on pavlovian and instrumental aversive learning.

Nevertheless, we propose that our results reflect greater specificity of the role of serotonin in behavioral inhibition than has been demonstrated previously in animals or humans. In essence, we have shown that serotonin is critical for linking behavioral inhibition with predictions of aversive outcomes rather than performing inhibitory or aversive processing alone. Our findings provide the first empirical support for an updated theory of serotonin function, which implicates serotonin in aversive predictions (Dayan and Huys, 2009).

\section{References}

Ardis TC, Cahir M, Elliott JJ, Bell R, Reynolds GP, Cooper SJ (2009) Effect of acute tryptophan depletion on noradrenaline and dopamine in the rat brain. J Psychopharmacol 23:51-55.

Aron AR, Robbins TW, Poldrack RA (2004) Inhibition and the right inferior frontal cortex. Trends Cogn Sci 8:170-177.

Avila C (2001) Distinguishing BIS-mediated and BAS-mediated disinhibition mechanisms: a comparison of disinhibition models of Gray $(1981,1987)$ and of Patterson and Newman (1993). J Pers Soc Psychol 80:311-324.

Bari A, Eagle DM, Mar AC, Robinson ES, Robbins TW (2009) Dissociable effects of noradrenaline, dopamine, and serotonin uptake blockade on stop task performance in rats. Psychopharmacology (Berl) 205:273-283.

Biggio G, Fadda F, Fanni P, Tagliamonte A, Gessa GL (1974) Rapid depletion of serum tryptophan, brain tryptophan, serotonin and 5-hydroxyindoleacetic acid by a tryptophan-free diet. Life Sci 14:1321-1329.

Browning M, Reid C, Cowen PJ, Goodwin GM, Harmer CJ (2007) A single dose of citalopram increases fear recognition in healthy subjects. J Psychopharmacol 21:684-690.

Carpenter LL, Anderson GM, Pelton GH, Gudin JA, Kirwin PD, Price LH, Heninger GR, McDougle CJ (1998) Tryptophan depletion during continuous CSF sampling in healthy human subjects. Neuropsychopharmacology 19:26-35. 
Cervo L, Mocaër E, Bertaglia A, Samanin R (2000) Roles of 5-HT(1A) receptors in the dorsal raphe and dorsal hippocampus in anxiety assessed by the behavioral effects of 8-OH-DPAT and S 15535 in a modified GellerSeifter conflict model. Neuropharmacology 39:1037-1043.

Chamberlain SR, Müller U, Blackwell AD, Clark L, Robbins TW, Sahakian BJ (2006) Neurochemical modulation of response inhibition and probabilistic learning in humans. Science 311:861-863.

Clark L, Roiser JP, Cools R, Rubinsztein DC, Sahakian BJ, Robbins TW (2005) Stop signal response inhibition is not modulated by tryptophan depletion or the serotonin transporter polymorphism in healthy volunteers: implications for the 5-HT theory of impulsivity. Psychopharmacology (Berl) 182:570-578.

Cochran WG, Cox GM (1957) Experimental designs. New York: Wiley.

Cools R, Blackwell A, Clark L, Menzies L, Cox S, Robbins TW (2005) Tryptophan depletion disrupts the motivational guidance of goal-directed behavior as a function of trait impulsivity. Neuropsychopharmacology 30:1362-1373.

Cools R, Robinson OJ, Sahakian B (2008a) Acute tryptophan depletion in healthy volunteers enhances punishment prediction but does not affect reward prediction. Neuropsychopharmacology 33:2291-2299.

Cools R, Roberts AC, Robbins TW (2008b) Serotoninergic regulation of emotional and behavioural control processes. Trends Cogn Sci 12:31-40.

Daw ND, Kakade S, Dayan P (2002) Opponent interactions between serotonin and dopamine. Neural Netw 15:603-616.

Dayan P, Huys QJ (2009) Serotonin in affective control. Annu Rev Neurosci 32:95-126.

Dekeyne A, Brocco M, Adhumeau A, Gobert A, Millan MJ (2000) The selective serotonin (5-HT) 1A receptor ligand, S15535, displays anxiolyticlike effects in the social interaction and Vogel models and suppresses dialysate levels of 5-HT in the dorsal hippocampus of freely-moving rats. A comparison with other anxiolytic agents. Psychopharmacology (Berl) 152:55-66.

Eagle DM, Bari A, Robbins TW (2008) The neuropsychopharmacology of action inhibition: cross-species translation of the stop-signal and go/ no-go tasks. Psychopharmacology (Berl) 199:439-456.

Eagle DM, Lehmann O, Theobald DE, Pena Y, Zakaria R, Ghosh R, Dalley JW, Robbins TW (2009) Serotonin depletion impairs waiting but not stopsignal reaction time in rats: implications for theories of the role of 5-HT in behavioral inhibition. Neuropsychopharmacology 34:1311-1321.

Evers EA, van der Veen FM, Jolles J, Deutz NE, Schmitt JA (2006a) Acute tryptophan depletion improves performance and modulates the BOLD response during a Stroop task in healthy females. Neuroimage 32:248-255.

Evers EA, van der Veen FM, van Deursen JA, Schmitt JA, Deutz NE, Jolles J (2006b) The effect of acute tryptophan depletion on the BOLD response during performance monitoring and response inhibition in healthy male volunteers. Psychopharmacology (Berl) 187:200-208.

Fadda F, Cocco S, Stancampiano R (2000a) A physiological method to selectively decrease brain serotonin release. Brain Res Brain Res Protoc 5:219-222.

Fadda F, Cocco S, Rossetti ZL, Melis G, Stancampiano R (2000b) A tryptophan-free diet markedly reduces frontocortical 5-HT release, but fails to modify ethanol preference in alcohol-preferring (sP) and nonpreferring (sNP) rats. Behav Brain Res 108:127-132.

Geller I, Demarco AO, Seifter J (1960) Delayed effects of nicotine on timing behavior in the rat. Science 131:735-737.

Gessa GL, Biggio G, Fadda F, Corsini GU, Tagliamonte A (1974) Effect of the oral administration of tryptophan-free amino acid mixtures on serum tryptophan, brain tryptophan and serotonin metabolism. J Neurochem 22:869-870.

Graeff FG (2002) On serotonin and experimental anxiety. Psychopharmacology (Berl) 163:467-476.

Graeff FG, Schoenfeld RI (1970) Tryptaminergic mechanisms in punished and nonpunished behavior. J Pharmacol Exp Ther 173:277-283.

Gray JA, McNaughton N (2000) The neuropsychology of anxiety. Oxford: Oxford UP.

Harrison AA, Everitt BJ, Robbins TW (1999) Central serotonin depletion impairs both the acquisition and performance of a symmetrically reinforced go/no-go conditional visual discrimination. Behav Brain Res 100:99-112.

Killcross S, Robbins TW, Everitt BJ (1997) Different types of fearconditioned behaviour mediated by separate nuclei within amygdala. Nature 388:377-380.

Klaassen T, Riedel WJ, Deutz NE, Van Praag HM (2002) Mood congruent memory bias induced by tryptophan depletion. Psychol Med 32:167-172.
LeDoux JE (1996) The emotional brain. New York: Simon and Schuster.

LeMarquand DG, Pihl RO, Young SN, Tremblay RE, Séguin JR, Palmour RM, Benkelfat C (1998) Tryptophan depletion, executive functions, and disinhibition in aggressive, adolescent males. Neuropsychopharmacology 19:333-341.

LeMarquand DG, Benkelfat C, Pihl RO, Palmour RM, Young SN (1999) Behavioral disinhibition induced by tryptophan depletion in nonalcoholic young men with multigenerational family histories of paternal alcoholism. Am J Psychiatry 156:1771-1779.

Moja EA, Cipolla P, Castoldi D, Tofanetti O (1989) Dose-response decrease in plasma tryptophan and in brain tryptophan and serotonin after tryptophan-free amino acid mixtures in rats. Life Sci 44:971-976.

Murphy FC, Smith KA, Cowen PJ, Robbins TW, Sahakian BJ (2002) The effects of tryptophan depletion on cognitive and affective processing in healthy volunteers. Psychopharmacology (Berl) 163:42-53.

Newman JP, Wallace JF, Schmitt WA, Arnett PA (1997) Behavioral inhibition system functioning in anxious, impulsive and psychopathic individuals. Pers Individ Dif 23:583-592.

Nishizawa S, Benkelfat C, Young SN, Leyton M, Mzengeza S, de Montigny C, Blier P, Diksic M (1997) Differences between males and females in rates of serotonin synthesis in human brain. Proc Natl Acad Sci U S A 94:5308-5313.

Rogers RD, Blackshaw AJ, Middleton HC, Matthews K, Hawtin K, Crowley C, Hopwood A, Wallace C, Deakin JF, Sahakian BJ, Robbins TW (1999) Tryptophan depletion impairs stimulus-reward learning while methylphenidate disrupts attentional control in healthy young adults: implications for the monoaminergic basis of impulsive behaviour. Psychopharmacology (Berl) 146:482-491.

Rubia K, Lee F, Cleare AJ, Tunstall N, Fu CH, Brammer M, McGuire P (2005) Tryptophan depletion reduces right inferior prefrontal activation during response inhibition in fast, event-related fMRI. Psychopharmacology (Berl) 179:791-803.

Soubrié P (1986) Reconciling the role of central serotonin neurones in human and animal behaviour. Behav Brain Res 9:319-364.

Stancampiano R, Melis F, Sarais L, Cocco S, Cugusi C, Fadda F (1997) Acute administration of a tryptophan-free amino acid mixture decreases 5-HT release in rat hippocampus in vivo. Am J Physiol 272:R991-R994.

Stanislaw H, Toderov N (1999) Calculation of signal detection theory measures. Behav Res Meth Instr Comp 31:137-149.

Stevens DA, Fechter LD, Resnick O (1969) The effects of p-chlorophenylalanine, a depletor of brain serotonin, on behavior. II. Retardation of passive avoidance learning. Life Sci 8:379-385.

Swets JA, Tanner WP Jr, Birdsall TG (1961) Decision processes in perception. Psychol Rev 68:301-340.

Takase LF, Nogueira MI, Baratta M, Bland ST, Watkins LR, Maier SF, Fornal CA, Jacobs BL (2004) Inescapable shock activates serotonergic neurons in all raphe nuclei of rat. Behav Brain Res 153:233-239.

Thiébot MH, Hamon M, Soubríe P (1982) Attenuation of induced-anxiety in rats by chlordiazepoxide: role of raphe dorsalis benzodiazepine binding sites and serotoninergic neurons. Neuroscience 7:2287-2294.

Tottenham N, Tanaka JW, Leon AC, McCarry T, Nurse M, Hare TA, Marcus DJ, Westerlund A, Casey BJ, Nelson C (2009) The NimStim set of facial expressions: judgments from untrained research participants. Psychiatry Res 168:242-249.

Tye NC, Everitt BJ, Iversen SD (1977) 5-Hydroxytryptamine and punishment. Nature 268:741-743.

Tye NC, Iversen SD, Green AR (1979) The effects of benzodiazepines and serotonergic manipulations on punished responding. Neuropharmacology 18:689-695.

van der Plasse G, Meerkerk DT, Lieben CK, Blokland A, Feenstra MG (2007) Lack of evidence for reduced prefrontal cortical serotonin and dopamine efflux after acute tryptophan depletion. Psychopharmacology (Berl) 195:377-385.

van der Stelt HM, Broersen LM, Olivier B, Westenberg HG (2004) Effects of dietary tryptophan variations on extracellular serotonin in the dorsal hippocampus of rats. Psychopharmacology (Berl) 172:137-144.

Wise CD, Berger BD, Stein L (1973) Evidence of -noradrenergic reward receptors and serotonergic punishment receptors in the rat brain. Biol Psychiatry 6:3-21.

Young SN, Smith SE, Pihl RO, Ervin FR (1985) Tryptophan depletion causes a rapid lowering of mood in normal males. Psychopharmacology (Berl) 87:173-177. 